Revista Española de Antropología Americana

ISSN: 0556-6533

https://doi.org/10.5209/REAA.63702

\title{
Composición, procedencia y tecnologías de producción de las teselas de turquesa del Monte Tláloc
}

\author{
Andrea Pérez Martínez ${ }^{1}$
}

Recibido: 4 de septiembre de 2018 / Aceptado: 31 de octubre de 2018

Resumen. La turquesa fue un mineral profundamente valorado en la época prehispánica, puesto que fue empleada para la creación de diversos objetos, como cuentas, pendientes e incluso pequeñas teselas que conformaban hermosos mosaicos. Este mineral ha sido recuperado en diferentes sitios; entre ellos, en el interior del templo prehispánico del Monte Tláloc donde fueron recuperadas miles de pequeñas teselas de turquesa. Dicho material fue analizado con diferentes técnicas no destructivas ni invasivas para su identificación precisa e inferir su procedencia. Asimismo, se analizaron las diferentes huellas de manufactura presentes en las piezas con el fin de identificar los procesos que se emplearon en la elaboración de las teselas y de, esta manera, asociar el material con algún estilo o tradición tecnológica.

Palabras clave: turquesa; yacimiento; manufactura; estilo tecnológico.

\section{[en] Composition, Provenance and Production Technologies of the Turquoise Tesserae of Monte Tlaloc}

\begin{abstract}
Turquoise was a mineral deeply valued in pre-Hispanic time; it was used to make different objects, such as beads, pendants and even small tesserae, which were used to design beautiful mosaics. This mineral has been recovered from different sites, such as the pre-Hispanic temple the Monte Tlaloc. Inside of this place were recovered thousands of small turquoise tesserae. These materials were analyzed with different non-destructive or invasive techniques for the precise identification of the material and infer their provenance. In addition, different manufacturing traces present in the pieces were analyzed in order to identify the processes that were used in the manufacture of the tesserae and associating the material with some style or technological tradition.
\end{abstract}

Keywords: turquoise; source; manufacturing; technological style.

Sumario. 1. Introducción. 2. Metodología de trabajo. 3. Identificación mineralógica. 4. Tecnologías de manufactura. 5. Discusión y conclusiones. 6. Referencias.

Cómo citar: Pérez Martínez, Andrea. 2018. «Composición, procedencia y tecnologías de producción de las teselas de turquesa del Monte Tláloc». Revista Española de Antropología Americana 48: 277290.

\section{Introducción}

El sitio arqueológico Monte Tláloc es un yacimiento localizado en la montaña homónima, de aproximadamente 4.120 msnm (INEGI 2013: 11), que se ubica en el Estado

1 Dirección de Estudios Arqueológicos, Instituto Nacional de Antropología e Historia, México. arqueologandrea@gmail.com 


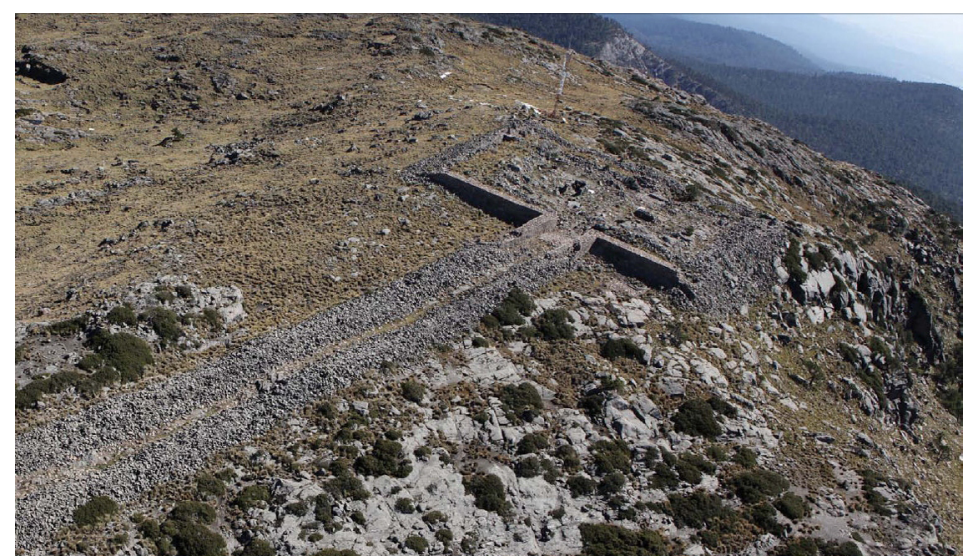

Figura 1. Templo dedicado a Tláloc en la cima de la montaña homónima. (Fotografía de Francisco Rentería Beltrán, 2012).

de México, dentro del municipio de Texcoco. En su cima se encuentra un templo prehispánico compuesto por una amplia calzada de aproximadamente $150 \mathrm{~m}$ de largo que remata en un cuadrángulo que en época prehispánica fue denominado como tetzacual$c o^{2}$, el cual posee un área de alrededor de $3000 \mathrm{~m}^{2}$ (Arribalzaga 2006: 15) (Figura 1).

Durante las temporadas de campo de 2006 y 2007 del Proyecto Arqueológico Cerro Tláloc (PACT), se realizó un recorrido sistemático dentro del tetzacualco, en donde se localizaron en superficie algunas teselas de turquesa; se calculó la extensión de la dispersión y se realizaron dos calas de $1 \times 3 \mathrm{~m}$, separadas por $2 \mathrm{~m}$ entre ellas, para la recuperación de los materiales. La profundidad promedio a la que se localizaron fue de $20 \mathrm{~cm}$ (Arribalzaga 2006: 15-16).

Uno de los primeros objetivos que se plantearon fue determinar cuál era la procedencia de la materia prima que se empleó para la elaboración de las teselas; para ello sería indispensable identificar la composición mineralógica del material. Es decir, identificar si las teselas del Monte Tláloc fueron manufacturadas con turquesa química o turquesa cultural. De acuerdo con la definición de Weigand (1993: 315), se denomina turquesa química a los minerales que geológica y químicamente coinciden con la composición de la verdadera turquesa: $\mathrm{Cu}(\mathrm{Al} 6 \mathrm{Fe})(\mathrm{PO} 4) 4(\mathrm{OH}) 8(4 \mathrm{H} 2 \mathrm{O})$ (Serrano 1980: 152). Por otro lado, la turquesa cultural comprende una amplia gama de minerales verde-azules que fueron empleados por las sociedades prehispánicas (malaquita, azurita, amazonita, crisocola, entre otras).

Otro de los objetivos era inferir los procesos de manufactura que se emplearon para la elaboración de las teselas, y con ello asociar el material con algún estilo o tradición tecnológica.

\section{Metodología de trabajo}

Uno de los primeros pasos del estudio de las teselas de turquesa fue un análisis tipológico, a partir del cual se pudo conocer la conformación total de la muestra. Poste-

\footnotetext{
2 Palabra náhuatl que significa «lugar encerrado de piedras».
} 
riormente, para el resto de los análisis, se realizó una selección de los objetos más representativos tomando en cuenta forma, color y contexto, entre otros atributos.

Con base en este primer análisis, se determinó que el total de la muestra se componía de 4.447 piezas, siendo la mayoría teselas de forma geométrica como cuadrángulos, rectángulos, triángulos, círculos, pentágonos, entre otros; aunque también se observan teselas no geométricas; asimismo, es importante señalar que se identificaron dos cuentas, dos piezas en proceso de manufactura y cuatro fragmentos de materia prima sin trabajar. Es muy probable que estos materiales conformaran un mosaico con iconografía, puesto que algunas teselas presentan líneas rectas bien definidas, e incluso se identificó la representación de dos pies con sandalias (Figura 2) y una pluma o punta de flecha, elementos muy similares a los atavíos que portan los personajes representados en el disco de mosaico de turquesa de la Ofrenda 99 del Templo Mayor de Tenochtitlán.

Debido a que se trata de objetos arqueológicos, se emplearon técnicas de identificación no invasivas ni destructivas, permitiendo, así, futuros análisis con otros métodos. Se seleccionaron cuatro técnicas para la caracterización del material: Fluorescencia de Luz Ultravioleta (UVF), Reflectografía Infrarroja (IR), Espectroscopía de energía dispersiva de rayos X o microanálisis por EDS y, finalmente, Espectroscopía Micro-Raman. Dichos métodos se usaron para la identificación de una muestra representativa y, en algunos casos, se aplicaron diferentes técnicas a una misma tesela para corroborar que los resultados fueran certeros.

Por otro lado, para la identificación de las técnicas de manufactura presentes en las teselas, se recurrió a la arqueología experimental, que se basa en reproducir, de la manera más similar posible, las tecnologías que se emplearon en el pasado para la realización de diferentes artefactos, con el fin de entender el comportamiento cultural de épocas pretéritas (Ascher 1961: 793). Con el apoyo del proyecto «Estilo y Tecnología de los objetos lapidarios en el México Antiguo», dirigido por el Dr. Emiliano Melgar, se realizaron diferentes experimentos en turquesa (desgastes, cortes, perforaciones, esgrafiados y acabados), para contrastar las huellas de manufactura con las teselas de turquesa del Monte Tláloc (Figura 3). A la hora de comparar los rasgos de las piezas se emplearon diferentes niveles de análisis, comenzando con la observación macroscópica, posteriormente con el Microscopio Estereoscópico (ME) a 10x y 30x y, finalmente, con el Microscopio Electrónico de Barrido (MEB), que permitió cumplir con los criterios de observación de 100x, 300x, 600x y 1000x planteados por Velázquez (2004) y Melgar (2014).
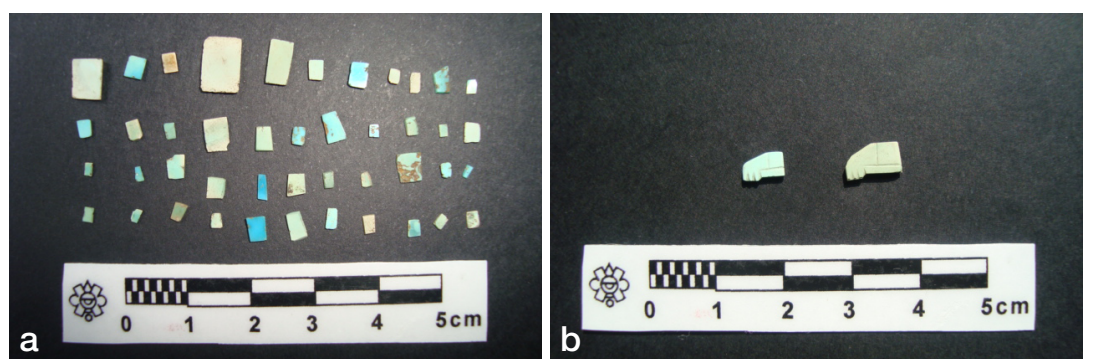

Figura 2. Teselas de la muestra analizada: a) de tipo rectangular; b) representación de parte antropomorfa (pie con sandalia). (Fotografías de Andrea Pérez, 2015). 

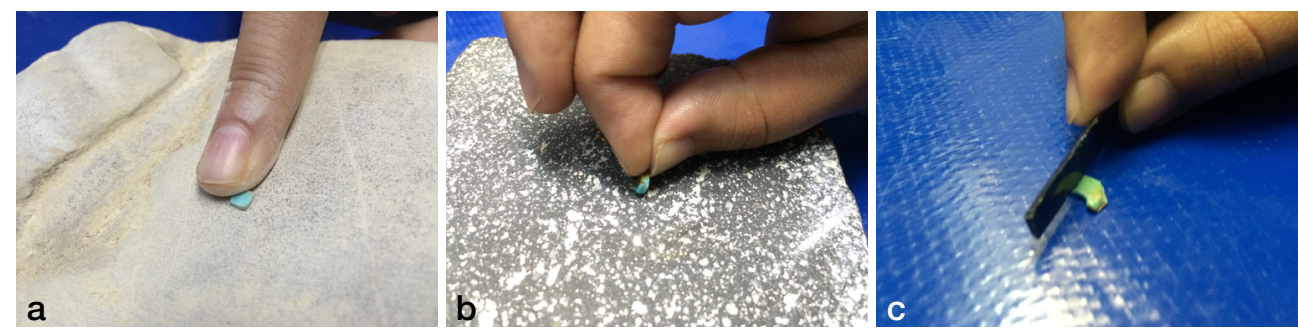

Figura 3. Arqueología experimental: a) desgaste de turquesa sobre laja de arenisca; b) desgaste de turquesa sobre laja de basalto; c) corte de tesela con lasca de obsidiana; d) bruñido de tesela con piel.

(Fotografías de Andrea Pérez, 2015).

\section{Identificación mineralógica}

En este apartado se aborda la identificación precisa del material a través de diferentes métodos y técnicas con objeto de asociar las teselas con sus posibles fuentes de extracción.

\subsection{Fluorescencia de Luz Ultravioleta (UVF)}

En el caso de la turquesa, se ha observado que, al ser sometida a la luz ultravioleta tanto en onda corta $(250 \mathrm{~nm})$ como en onda larga $(365 \mathrm{~nm})$, emite distintas coloraciones, como las azules y café, que coinciden con turquesas químicas (Schneider 2006: 89), mientras que la turquesa cultural presenta colores opacos y oscuros, e incluso rosa en el caso de la amazonita (Melgar 2014: 193). La aplicación de esta técnica a las poco más de trescientas teselas de la muestra dio como resultado la fluorescencia de todas ellas (Figura 4), por lo que se puede concluir que coinciden con las muestras de referencia expuestas por Melgar (2014: 193), en cuyo trabajo se refiere que la materia prima coincide con la turquesa química procedente del Suroeste de los Estados Unidos y el Noroeste de México.

\subsection{Reflectografía Infrarroja}

La reflectografía infrarroja expone los materiales a luz infrarroja para observar su opacidad o grado de reflejo de la luz, permitiendo diferenciar ciertos minerales (Ruvalcaba et al. 2010: 156). Tal como se puede observar en la Figura 5, las teselas en luz visible son azules, pero, al aplicarles el infrarrojo, se observan en color grisáceo claro, lo cual implica una alta reflectividad. Este dato hace posible asociar el mineral con turquesa verdadera. No obstante, el hecho de que se perciban diferencias de tonos entre las teselas demuestra que, si bien son turquesas químicas, no todas ellas proceden de un mismo yacimiento. 


\subsection{Espectroscopia de energía dispersiva de rayos $\mathrm{X}$ o microanálisis por EDS}

La espectroscopia de energía dispersiva de rayos $\mathrm{X}$ se basa en un detector de ese tipo de energía, el cual se encuentra instalado en la cámara de análisis del Microscópico Electrónico de Barrido y permite obtener imágenes y espectros de composición química elemental (Kang et al. 2013: 663). Con este método se analizaron poco más de ciento cincuenta teselas, algunas de ellas ya analizadas con las técnicas antes expuestas; como ha quedado señalado más arriba, se decidió aplicar diferentes metodologías de identificación a las mismas piezas para asegurar que en realidad se estaba trabajando con turquesa química.
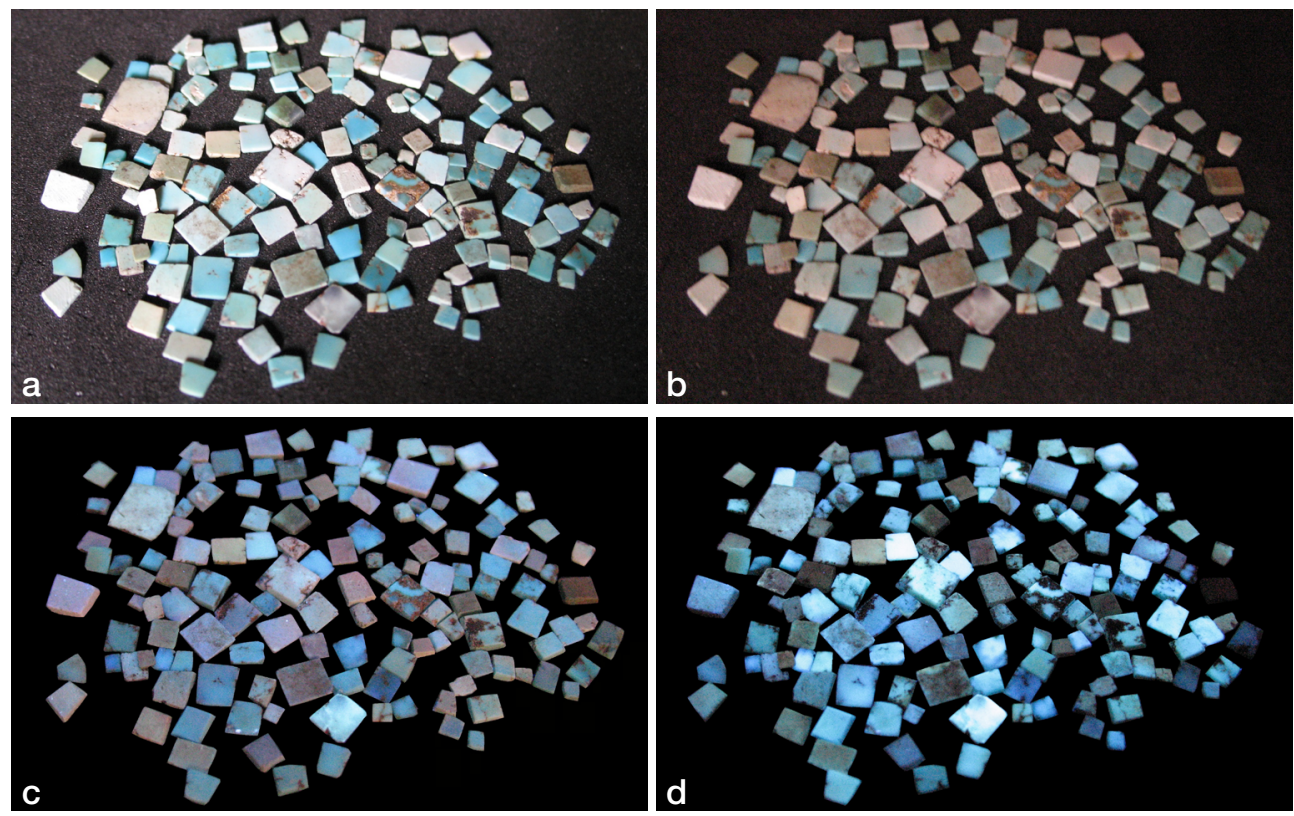

Figura 4. Análisis de teselas de turquesa de tipo cuadrangular: a) con luz visible; b) ambiente oscuro; c) irradiación de luz ultravioleta banda corta; d) banda larga.

(Fotografías de Emiliano Melgar, 2015).
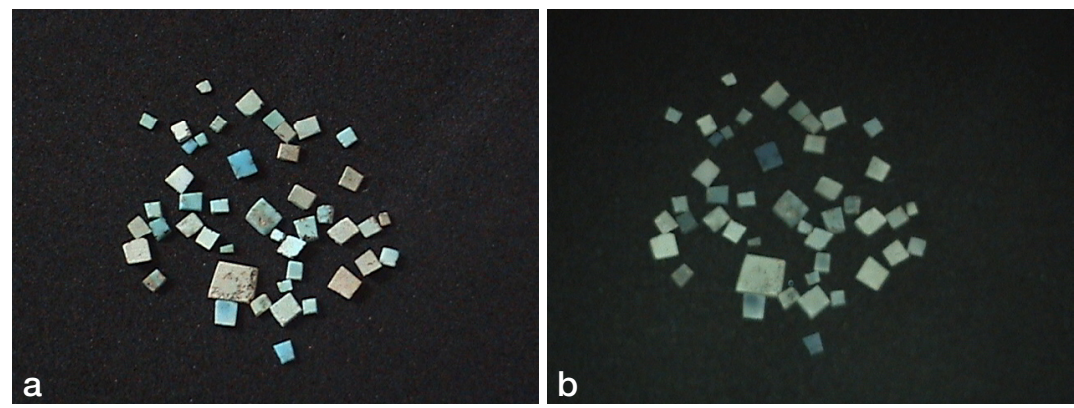

Figura 5. Análisis de teselas de turquesa de tipo cuadrangular: a) con luz visible;

b) irradiación de luz infrarroja. (Fotografías de Emiliano Melgar, 2015). 
Basándonos en los resultados obtenidos, está claro que las teselas halladas en la cima del Monte Tláloc son turquesas químicas o verdaderas. Sin embargo, al comparar cada uno de los espectros, se observa que -a pesar de compartir una misma fórmula química-no son idénticos; en algunos de ellos se identifica la presencia de ciertas trazas de hierro ( $\mathrm{Fe}$ ), silicio ( $\mathrm{Si}$ ) y zinc ( $\mathrm{Zn}$ ), entre otras (en muy bajos porcentajes). Esto apunta, de nuevo, a que la materia prima no fue extraída de un solo yacimiento.

Por tal razón, se decidió realizar microanálisis por EDS a muestras de referencia, con objeto de comparar los espectros de las teselas de turquesa con espectros de muestras obtenidas en diferentes yacimientos del Suroeste de los Estados Unidos y el Noroeste de México. De entre los elementos químicos presentes en la mayoría de la muestra de teselas, se prestó mayor atención al hierro $(\mathrm{Fe})$ y al cobre $(\mathrm{Cu})$ y dichos elementos se sometieron a análisis. Como se observa en las gráficas de la Figura 6, las muestras de referencia se dividieron en cuatro grupos ${ }^{3}$ : Nuevo México, Arizona, Nevada y Baja California, cada uno de los cuales se encuentra representado por diferentes yacimientos. La comparación entre las muestras de referencia y las teselas de turquesa evidencian la asociación de la mayoría de los materiales de éstas con las minas de Arizona -Sleeping Beauty, Kingman y Bisbee-, así como algunos yacimientos de Nuevo México -Tyrone, Santa Rita y Cerrillos-.
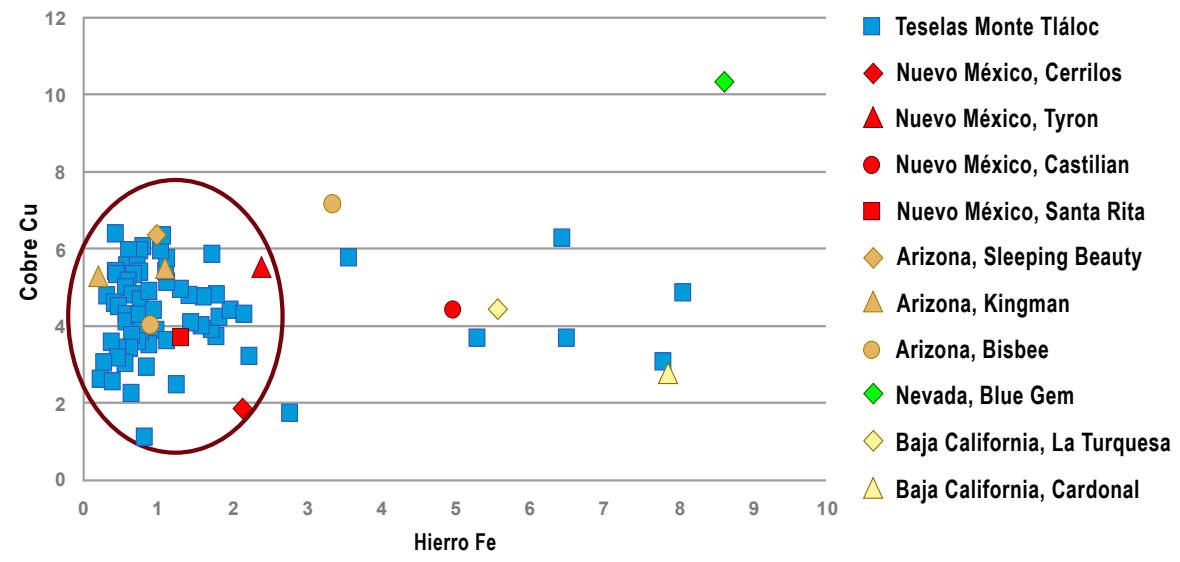

Figura 6. Contrastación hierro/cobre mostrando la asociación de los materiales procedentes del Monte Tláloc con diferentes minas.

(Elaboración de Andrea Pérez, 2016).

\subsection{Espectroscopia Micro-Raman}

La espectroscopia Raman es una técnica fotónica de alta resolución, en la cual un haz intenso de luz monocromática pasa a través de la muestra; el análisis se basa en la cantidad de luz dispersada por el material. Este método proporciona información química y estructural en pocos segundos, haciendo posible la identificación de los arreglos moleculares de los compuestos (Castellan 1987: 672). Al realizar el análisis a las teselas

\footnotetext{
3 Cabe señalar que también se efectuaron análisis a muestras de referencia procedentes de la mina de Cumobabi, Sonora; sin embargo, dichas muestras carecen de Hierro $(\mathrm{Fe})$ y Calcio $(\mathrm{Ca})$, por lo que no aparecen representadas en la gráfica.
} 
de turquesa, una vez más se corroboró que el material con el que fueron elaboradas corresponde a turquesa química o verdadera. En comparación con el análisis de EDS, con el Micro-Raman no se identifican los elementos químicos, sino que se distinguen las vibraciones moleculares de los compuestos que conforman la muestra (Figura 7). Una vez obtenidos los espectros de las teselas de turquesa, al igual que en el caso anterior, se realizaron comparaciones con diferentes muestras de referencia (Figura 8), pudiendo comprobarse que la mayoría de las teselas corresponden a dos de los yacimientos de Arizona que sobresalieron aplicando la técnica de EDS: Kingman y Bisbee.

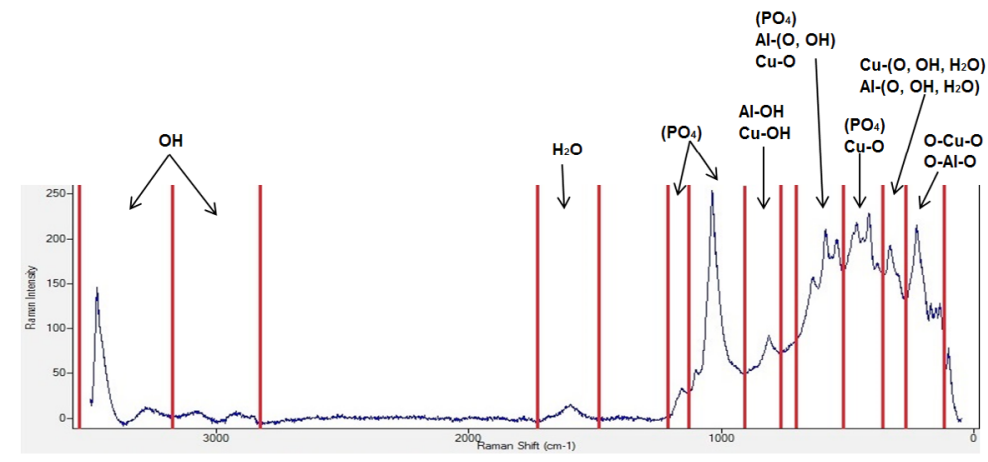

Figura 7. Espectro de tesela de tipo rectangular, con indicación de los rangos de los diferentes compuestos presentes en la turquesa basados en Frost et al. (2006) y Čejka et al. (2015). (Espectro de Cristina

Zorrilla, 2016. Elaboración de Andrea Pérez, 2016).
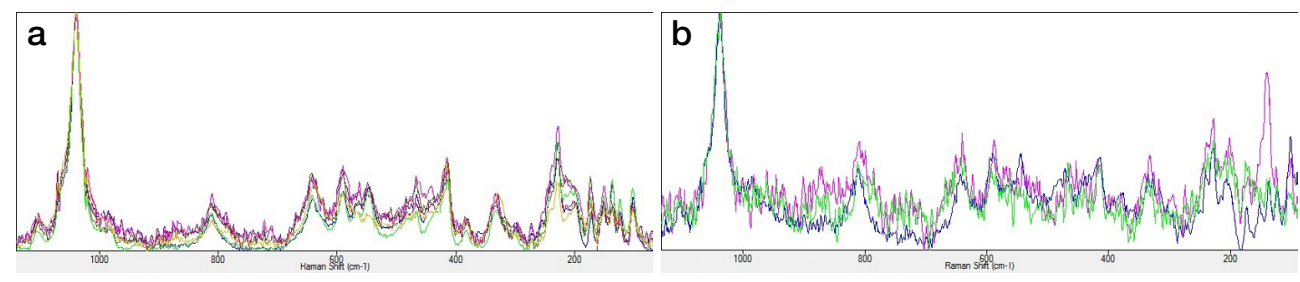

Figura 8. Espectros de turquesas procedentes de la mina de Kingman, Arizona (a) y de la mina de Bisbee, Arizona (b); se observa que diferentes teselas de turquesa del Monte Tláloc se ajustan a las mismas señales. (Espectros de Cristina Zorrilla, 2016).

\section{Tecnologías de manufactura}

Una vez identificadas las teselas con las técnicas antes descritas, se procedió a su análisis con el empleo del Microscopio Estereoscópico y el Microscopio Electrónico de Barrido, comparando las huellas de las piezas arqueológicas con las experimentales anteriormente realizadas y obteniéndose los siguientes resultados.

\subsection{Desgastes}

Se analizaron los desgastes presentes en diferentes teselas de turquesa con el Microscopio Estereoscópico y fue posible apreciar una gran cantidad de rayones rectos 
hacia diferentes direcciones, tal como se presenta en la Figura 9, lo cual sugiere que la materia prima fue frotada contra una laja o un metate sin el empleo de un abrasivo. Al observar las micrografías obtenidas con el MEB, se identificaron dos patrones: el primero se caracteriza por presentar bandas rectas y entrecruzadas de $10 \mu \mathrm{m}$ de espesor, producto de la aglomeración de líneas muy finas que van de 3 a 3,5 $\mu \mathrm{m}$; ello corresponde a las huellas producidas por la arenisca (Melgar 2014: 253) (Figura 10). El segundo patrón corresponde a las superficies desgastadas con basalto, el cual plasma sobre la superficie de las teselas bandas bien definidas con un grosor de 100 $\mu \mathrm{m}$ (Melgar 2014: 252) (Figura 11).
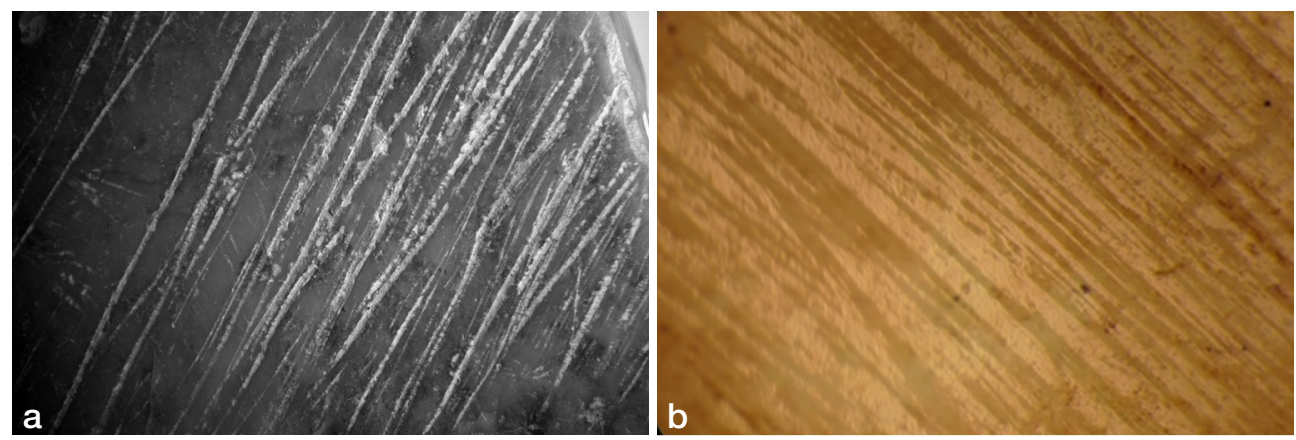

Figura 9. a) Desgaste experimental sin empleo de abrasivo; b) superficie de tesela pentagonal. Amplicación: 30x. (Fotografías de Emiliano Melgar, 2016).
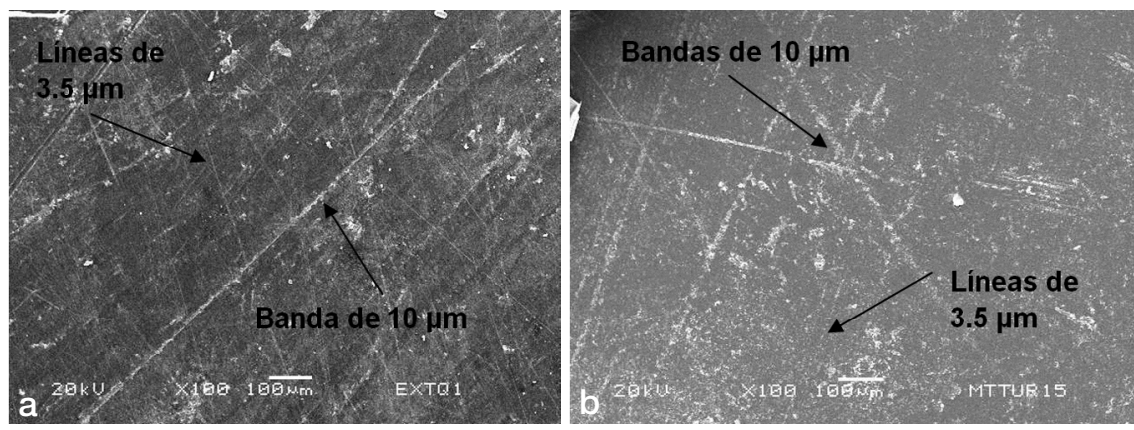

Figura 10. a) Desgaste experimental con arenisca; b) superficie de tesela cuadrangular. Ampliación: 100x. (Micrografías de Gerardo Villa, 2015).
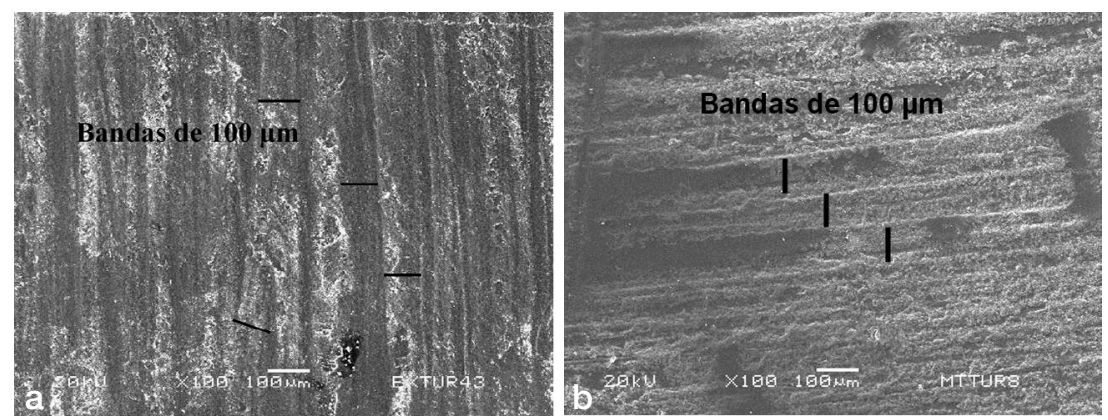

Figura 11. a) Desgaste experimental con basalto; b) tesela cuadrangular. Ampliación: 100x. (Micrografías de Gerardo Villa, 2015). 


\subsection{Cortes}

En el caso de los cortes, la comparación entre las piezas arqueológicas y las experimentales identificó dos patrones. El primero corresponde a los cortes realizados con lascas de obsidiana (Figura 12); dicha herramienta genera líneas finas rectas y espaciadas de 0,6 a $1,3 \mu \mathrm{m}$ de grosor, las cuales -en muchas ocasiones- se aglomeran y forman una textura rugosa (Melgar 2014: 257). Es interesante señalar que el segundo patrón de manufactura fue identificado no propiamente como un corte, sino como un desgaste; al realizar los experimentos se observó que, dado el diminuto tamaño de las teselas, es probable que para generar bordes tan lisos se haya optado por el desgaste; tal como se muestra en la Figura 13, las huellas coinciden con la arenisca (bandas de 10 $\mu \mathrm{m}$ de espesor producto de la aglomeración de líneas muy finas que van de 3 a 3,5 $\mu \mathrm{m}$ ).
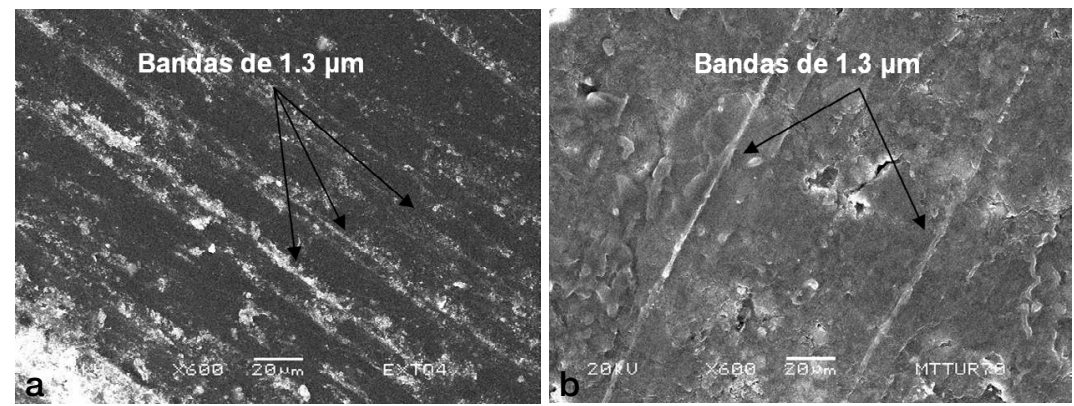

Figura 12. a) Corte experimental con lasca de obsidiana b) Borde de tesela pentagonal. Ampliación: 600x. (Micrografías de Gerardo Villa, 2015).
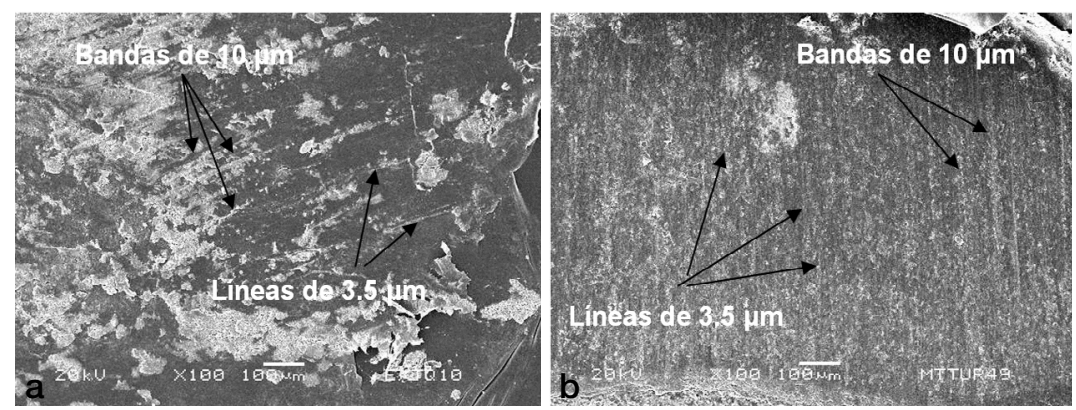

Figura 13. a) Desgaste experimental con arenisca b) Borde de tesela cuadrangular. Ampliación: 100x. (micrografías: Gerardo Villa, 2015).

\subsection{Perforaciones}

Sólo observamos perforaciones en tres piezas del total de la muestra. En las paredes de cada una de las perforaciones se perciben líneas muy finas, casi imperceptibles, lo cual coincide con los experimentos en donde se emplea un abrasivo (arena, polvo de obsidiana, polvo de pedernal, ceniza volcánica u otro) con la ayuda de un carrizo (Figura 14). Al analizar dichas piezas con el MEB, se identificó que las perforaciones 
fueron elaboradas a partir del empleo de polvo de pedernal, puesto que en las huellas se observa una superficie rugosa con textura rugosa, así como líneas finas de $1 \mu \mathrm{m}$ de ancho (Melgar 2014: 369) (Figura 15).
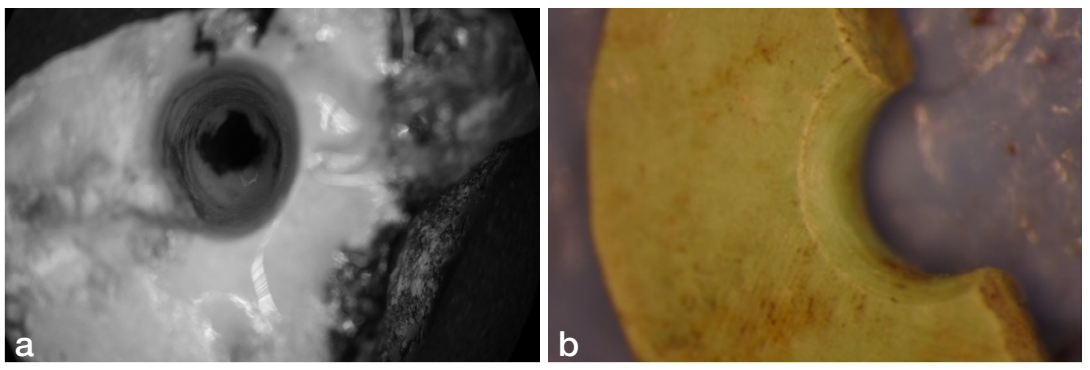

Figura 14. a) Perforación experimental con polvo de pedernal y carrizo, 10x; b) perforación de fragmento de cuenta, 30x. (Fotografías de Emiliano Melgar, 2016).
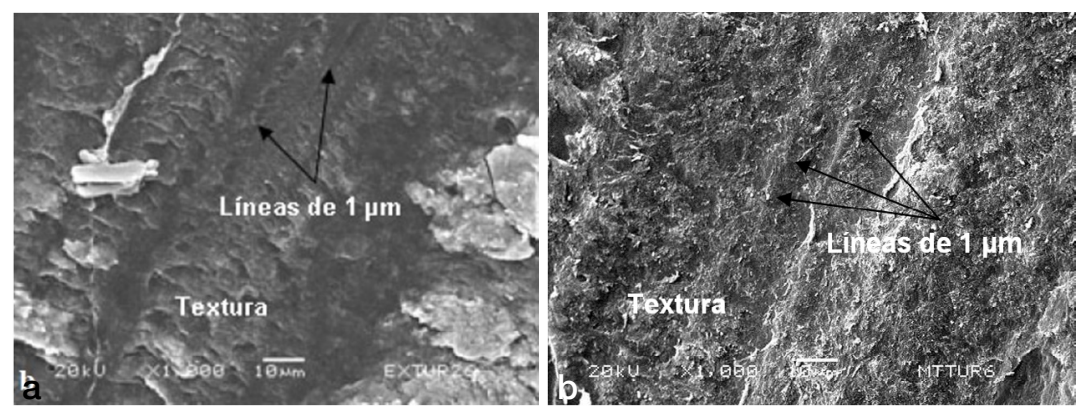

Figura 15. a) Perforación experimental con polvo de pedernal y carrizo; b) horadación de cuenta. Ampliación: 1000x.

(Micrografías de Gerardo Villa, 2015).

\subsection{Esgrafiados}

Para determinar la herramienta lítica que se empleó en la elaboración de esgrafiados, se analizaron diferentes micrografías. En ellas son notorias líneas finas y rectas con un grosor que va de 0,6 a 1,3 $\mu \mathrm{m}$, las cuales en muchas ocasiones se aglomeran y conforman una textura rugosa; dichos rasgos corresponden a las modificaciones realizadas con obsidiana (Figura 16) (Melgar 2014: 263).

\subsection{Acabados}

A la gran mayoría de las teselas se les aplicó un acabado, esto es: un último tratamiento tras el desgaste de regularización para obtener en una de las caras una superficie lustrosa, como la que se observa en la Figura 17. Al analizar las micrografías, se identificó que las incrustaciones carecen de pulido, por lo que se concluye que sólo fueron bruñidas con un trozo de piel; al estudiar las piezas desgastadas tanto en arenisca como basalto, resulta notorio que la aplicación de esta técnica aplana las bandas y hace que se observen áreas alisadas (Melgar 2014: 267) (Figuras 18 y 19). 

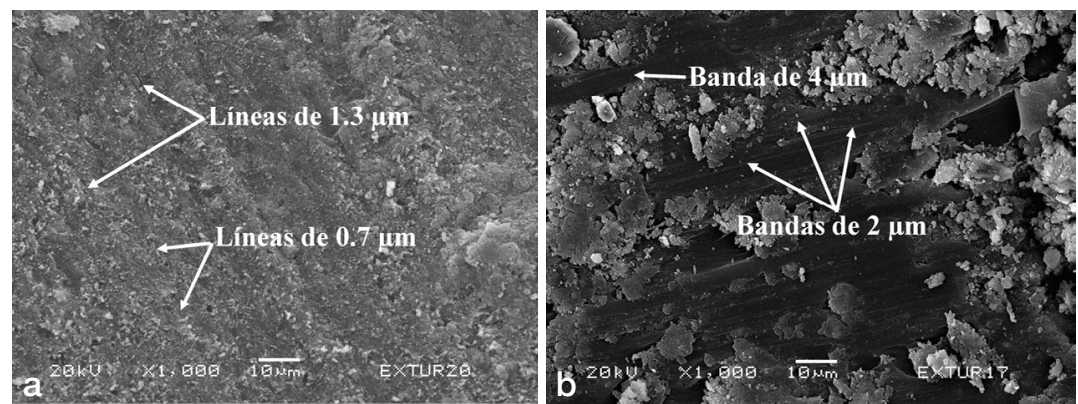

Figura 16. a) Esgrafiado experimental con lascas de obsidiana; b) esgrafiado en fragmento de tesela rectangular. Ampliación: 1000x. (Micrografías de Emiliano Melgar, 2014).
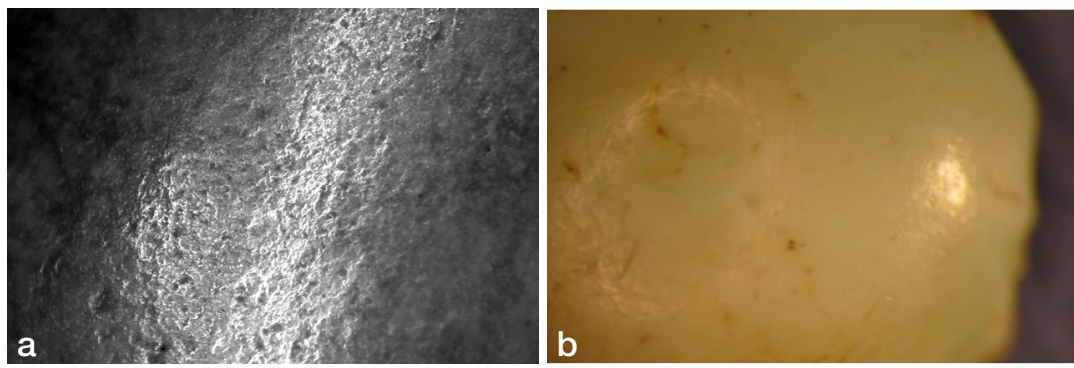

Figura 17. a) Acabado experimental bruñido con piel; b) superficie de cabochon. Ampliación: 30x. (Fotografías Emiliano Melgar, 2016).
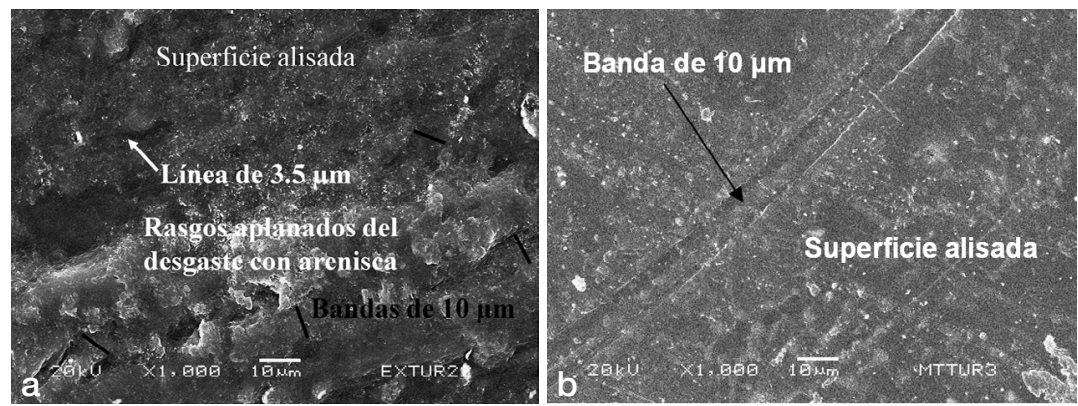

Figura 18. a) Acabado experimental bruñido con piel sobre desgaste con arenisca; b) superficie de pieza reutilizada. Ampliación: 1000x. (Micrografías de Gerardo Villa, 2015).
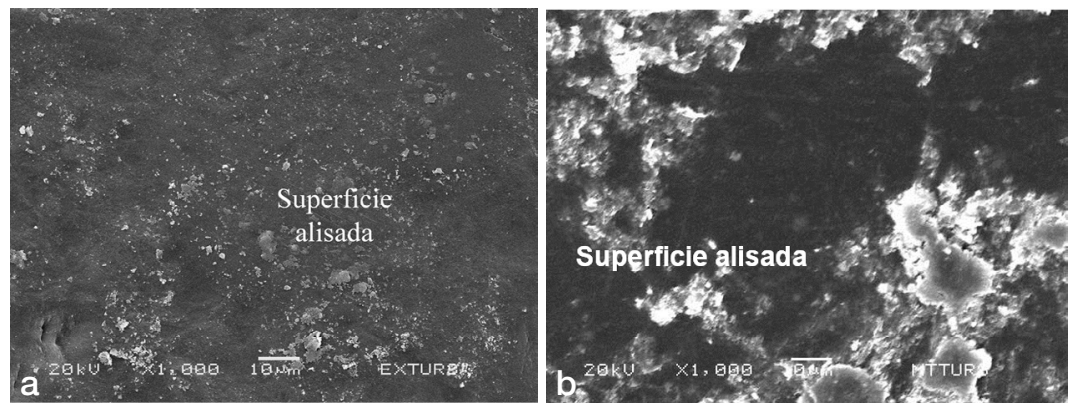

Figura 19. a) Acabado experimental bruñido con piel sobre desgaste con basalto; b) superficie de tesela cuadrangular. Ampliación: 1000x. (Micrografías de Gerardo Villa, 2015). 


\section{Discusión y conclusiones}

Basándonos en la combinación de las diferentes técnicas de identificación de materiales, se corroboró que todas las teselas analizadas coincidieron con la turquesa química o turquesa verdadera, $\mathrm{Cu}(\mathrm{Al} 6 \mathrm{Fe})(\mathrm{PO} 4) 4(\mathrm{OH}) 8(4 \mathrm{H} 2 \mathrm{O})$ (Serrano 1980: 152); podemos definir, pues, el material como un fosfoaluminato de cobre hidratado. La correcta identificación del material ha hecho posible asociarlo a ciertas áreas de extracción o yacimientos posibles, los cuales se localizan en el actual Suroeste de los Estados Unidos y el Noroeste de México, principalmente en Arizona (Sleeping Beauty, Kingman y Bisbee) y Nuevo México (Tyron, Santa Rita y Cerrillos).

Es evidente que la turquesa recuperada en el Monte Tláloc presenta una gran variabilidad en cuanto a su composición química; ello indica que la materia prima para la elaboración de las teselas no proviene de un único yacimiento. Es probable que esto se deba a cambios sociales ocurridos en las áreas de explotación, como, por ejemplo, el colapso de los grandes centros regionales que monopolizaban la distribución de los materiales (Melgar 2014: 273).

El templo prehispánico del Monte Tláloc fue concebido como un lugar de culto para la realización de diferentes ceremonias. Aun no tratándose de un contexto de taller o de un área de producción, fue posible determinar la secuencia operativa del material de estudio y, asimismo, asociarlo con diferentes estilos tecnológicos, los cuales se describen a continuación.

La mayoría de las teselas (81\%) destacan por presentar bajo el microscopio estereoscópico una superficie rayada en la que, al ser analizada a mayores amplificaciones, fue identificado el empleo de arenisca para regularizar tanto las caras como las paredes. En cuanto al acabado, éstas sólo mostraban huellas propias del bruñido con piel. Dichas técnicas coinciden con las huellas identificadas en talleres y sitios del Suroeste de los Estados Unidos, tales como el Cañón del Chaco (Posclásico Temprano) o Pottery Mound y Grasshopper Pueblo (Posclásico Tardío) (Melgar 2014: 293, 2016).

Por otro lado, se identificó otro grupo de huellas, las cuales se caracterizan por el empleo de lajas o metates de basalto para el desgaste, cortes y esgrafiados con obsidiana, perforaciones con polvo de pedernal y bruñido con piel en los acabados. Estas técnicas coinciden con lo que se ha denominado como «estilo tecnológico imperial tenochca», es decir, objetos creados por parte de la elite mexica para diferentes ceremonias y rituales, el cual se relaciona con la consolidación de la Tripe Alianza (Melgar 2014: 301).

La identificación de estos dos estilos tecnológicos indica que una parte mayoritaria de las teselas de turquesa del Monte Tláloc llegaron como productos terminados desde el Suroeste de los Estados Unidos, mientras que otras fueron elaboradas en talleres palaciegos mexicas. Es importante añadir que se reconoció, asimismo, la combinación de ambos estilos en la manufactura de algunas teselas, concretamente las que formaban parte de la representación, en el mosaico, de dos partes antropomorfas (pies con sandalias) y la pluma o punta de flecha. En este caso, se infiere que los primeros procesos de la cadena operativa se efectuaron en el Suroeste de los Estados Unidos, donde se obtuvo la materia prima, y lo más probable es que la misma fuera desgastada con arenisca para obtener una especie de preformas de teselas -con caras desgastadas y bordes sin trabajar-, de modo que fueran los consumidores finales quienes decidieran la forma específica de las teselas en la conformación de diferentes mosaicos (Melgar 2016). 
Cabe mencionar, por último, que este estudio constituye sólo una primera aproximación a los materiales lapidarios ofrendados en el templo prehispánico del Monte Tláloc; se pretende continuar con el análisis de los materiales para comprender mejor este interesante yacimiento.

Agradecimientos: Esta investigación no hubiera sido posible sin el apoyo del doctor Emiliano Melgar, director del proyecto «Estilo y Tecnología de los objetos lapidarios en el México Antiguo»; del doctor Gerardo Villa del laboratorio de Microscopía Electrónica del INAH; de la doctora Cristina Zorrilla, del laboratorio de Materiales Avanzados del Instituto de Física de la UNAM; del maestro Víctor Arribalzaga, director del Proyecto Arqueológico Monte Tláloc y de los integrantes del Taller de Arqueología Experimental en Lapidaria.

\section{Referencias}

Arribalzaga Tobón, Víctor Manuel. 2006. Informe parcial temporada de campo 2005; Prospección arqueológica y Propuesta de investigación arqueológica para desarrollarse en la cumbre del Cerro Tláloc, Estado de México, temporada de campo 2006. Archivo Técnico de la Coordinación Nacional de Arqueología, Instituto Nacional de Antropología e Historia, México.

Ascher, Robert. 1961. «Experimental Archaeology». American Anthropologist 63 (4): 793 816.

Castellan, Gilbert. 1987. Fisicoquímica. México: Pearson Education.

Instituto Nacional de Estadística y Geografía (INEGI). 2013. Perspectiva estadística: México. México: Instituto Nacional de Estadística y Geografía.

Kang, Kyung W., Juan M. Echarri y Carlos L. Llorente. 2013. «Servicio de microscopía electrónica de barrido y microanálisis del LIMF- Facultad de Ingeniería», en Segundas Jornadas de Investigación y Transferencia, pp. 660-665. La Plata: Universidad Nacional de la Plata.

Melgar Tísoc, Emiliano Ricardo. 2014. Comercio, tributo y producción de las turquesas del Templo Mayor de Tenochtitlán. Tesis doctoral. México: Universidad Nacional Autónoma de México.

—. 2016. «Las turquesas del Templo Mayor de Tenochtitlan y su relación con las tradiciones lapidarias de Mesoamérica, el Noroeste de México y el Suroeste de los Estados Unidos». Ponencia presentada en Seminario Permanente Taller Signos de Mesoamérica, México: Universidad Nacional Autónoma de México.

Ruvalcaba Sil, José Luis, Laura Filloy Nadal, Marina Vaggi, Luisa H. Tapia Gálvez y Rigoberto Sánchez Becerra. 2010. «Estudio no destructivo in situ de la máscara de Malinaltepec», en La máscara de Malinaltepec, Sofía Martínez del Campo, coord., pp. 153-168. México: Conaculta-Instituto Nacional de Antropología e Historia.

Schneider, Stuart. 2006. The World of Fluorescent Minerals. Atglen: Schiffer Publishing Ltd. Serrano, Mercé. 1980. Guía de minerales y rocas. Barcelona: Ediciones Grijalbo.

Velázquez Castro, Adrián. 2004. Técnicas de manufactura de los objetos de concha del Templo Mayor de Tenochtitlan. La producción especializada de los objetos de concha del Templo Mayor de Tenochtitlan. Tesis doctoral. México: Universidad Nacional Autónoma de México. 
Weigand, Phil C. 1993. «La función de la turquesa en la estructura comercial de la antigua Mesoamérica», en Evolución de una civilización prehispánica: arqueología de Jalisco, Nayarit y Zacatecas, Phil C. Weigand, pp. 313-341. Morelia: El Colegio de Michoacán. 